\title{
Wavelet based detection of ventricular arrhythmias with neural network classifier
}

\author{
Sankara Subramanian Arumugam ${ }^{1}$, Gurusamy Gurusamy ${ }^{2}$, Selvakumar Gopalasamy ${ }^{3}$
}

\footnotetext{
${ }^{1}$ Department of Electrical and Electronics Engineering, NPA Centenary Polytechnic College, Kothagiri, Tamilnadu, India; ${ }^{2}$ Department of Electrical and Electronics Engineering, Bannariamman Institute of Technology, Sathyamangalam, Tamilnadu, India; ${ }^{3}$ Department of Electrical Sciences, Vinayaka Mission's Kirupananda Variyar Engineering College, Salem, Tamilnadu, India. Email: sankararu@yahoo.com; ngselva kumar@yahoo.com
}

Received 29 August 2009; revised 10 September 2009; accepted 15 September 2009.

\begin{abstract}
This paper presents an algorithm based on the wavelet decomposition, for feature extraction from the Electrocardiogram (ECG) signal and recognition of three types of Ventricular Arrhythmias using neural networks. A set of Discrete Wavelet Transform (DWT) coefficients, which contain the maximum information about the arrhythmias, is selected from the wavelet decomposition. These coefficients are fed to the feed forward neural network which classifies the arrhythmias. The algorithm is applied on the ECG registrations from the MIT-BIH arrhythmia and malignant ventricular arrhythmia databases. We applied Daubechies 4 wavelet in our algorithm. The wavelet decomposition enabled us to perform the task efficiently and produced reliable results.
\end{abstract}

Keywords: Daubechies 4 Wavelet; ECG; Feed Forward Neural Network; Ventricular Arrhythmias; Wavelet Decomposition

\section{INTRODUCTION}

The cardiac disorders which are life threatening are the ventricular arrhythmias such as Ventricular Tachycardia (VT), Ventricular Fibrillation (VFIB) and Ventricular Flutter (VFL). The classification of ECG into these different pathological disease categories is a complex task.

Successful classification is achieved by finding the characteristic shapes of the ECG that discriminate effectively between the required diagnostic categories. Conventionally, a typical heart beat is identified from the ECG and the component waves of the QRS, T and P waves are characterized using measurements such as magnitude, duration and area (Figure 1).

In an arrhythmia monitoring system or a defibrillator, it is important that the algorithm for detecting ECG ab- normalities should be reliable. The patient will be loosing a chance of treatment if the system is not able to detect the arrhythmia. Also a false positive detection will initiate a defibrillator to give improper therapeutic intervention. Both situations are linked with the patient's life.

An algorithm based on Wavelet Transform is quite efficient for the detection of ventricular arrhythmias compared to the Discrete Fourier Transform (DFT) since it permits analyzing a discrete time signal using frequency components. The DWT has been used for analyzing, decomposing and compressing the ECG signals [1].

The wavelet transforms make possible, the decomposition of a signal into a set of different signals of restricted frequency bands. Wavelet processing can be considered as a set of band pass filters [2]. Moreover, the discrete wavelet transform corresponds to a multiresolution analysis [3] which can reduce the redundancy of each filtered signal so that the processing algorithm can be applied effectively to a small data subset of the original signal $[4,5,6,7,8,9]$

A classification scheme is developed in which a feed forward neural network is used as a classification tool depending on the distinctive frequency bands of each arrhythmia. The Back Propagation (BP) algorithm allows experiential acquisition of input/output mapping

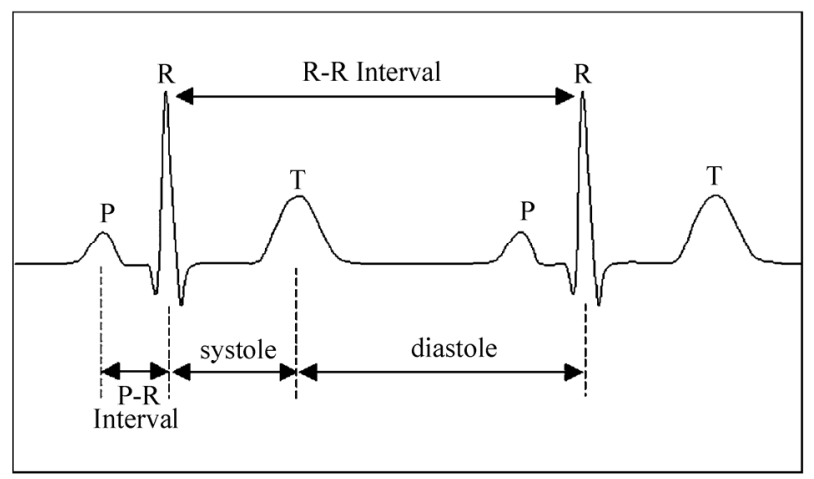

Figure 1. Normal ECG waveform. 
knowledge within multi-layer networks [10]. BP performs the gradient descent search to reduce the mean square error between the actual output of the network and the desired output through the adjustment of the weights. It is highly accurate for most classification problems because of the property of the generalized data rule. In the traditional BP training, the weights are adapted using a recursive algorithm starting at the output nodes and working back to the first hidden layer.

In our study, an algorithm is implemented to detect and classify Ventricular Tachycardia (VT), Ventricular Fibrillation (VFIB) and Ventricular Flutter (VFL) using wavelet decomposition and neural classification.

The ECG registrations from MIT-BIH arrhythmia and malignant ventricular arrhythmia databases are used here. The analysis is done using Daubechies 4 wavelet.

\section{VENTRICULAR ARRHYTHMIA}

Arrhythmias are the abnormal rhythms of the heart. They cause the heart to pump the blood less effectively. Most cardiac arrhythmias are temporary and benign. The ventricular arrhythmias are life threatening and need treatment. Such ventricular arrhythmias are Ventricular Tachycardia, Supraventricular Tachycardia, Ventricular Fibrillation and Ventricular Flutter.

VT is a difficult clinical problem for the physicians (Figure 2). Its evaluation and treatment are complicated because it often occurs in life-threatening situations that dictate rapid diagnosis and treatment. Ventricular tachycardia is defined as three or more beats of ventricular origin in succession at a rate greater than 100 beats/minute. There are no normal-looking QRS complexes. Because ventricular tachycardia originates in the ventricle, the QRS complexes on the electrocardiogram are widened ( $>0.12$ seconds). Ventricular tachycardia has the potential of degrading to the more serious ventricular fibrillation.

VFIB is a condition in which the heart's electrical activity becomes disordered (Figure 3). During Ventricular

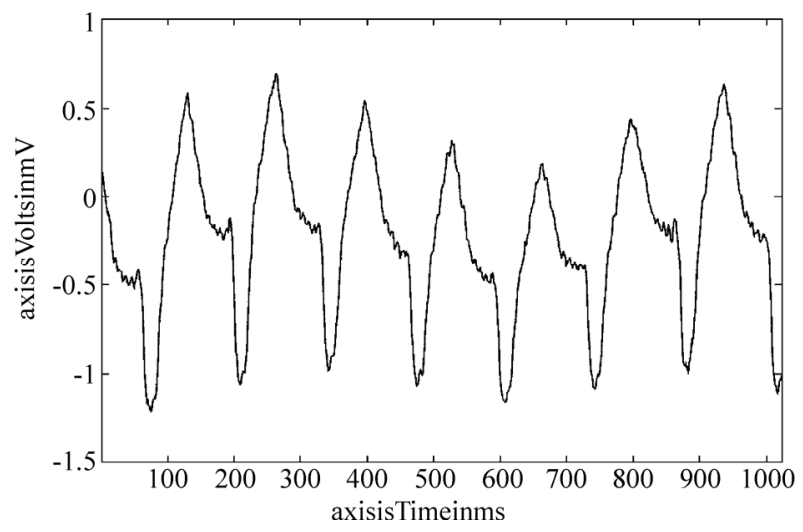

Figure 2. ECG waveform with VT.

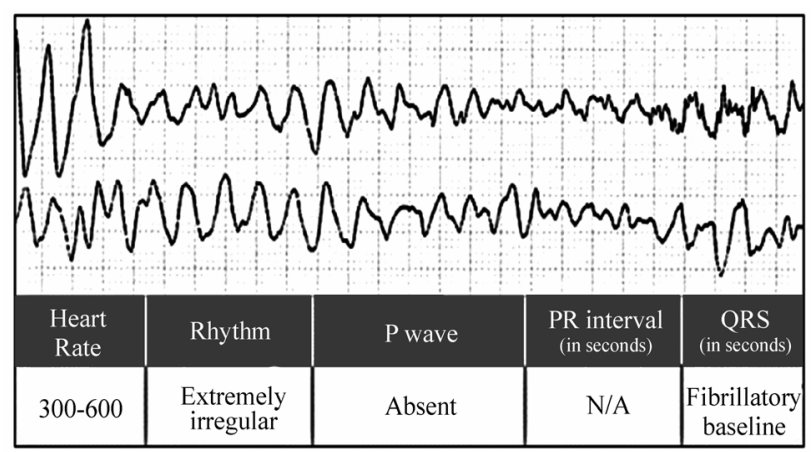

Figure 3. Ventricular fibrillation.

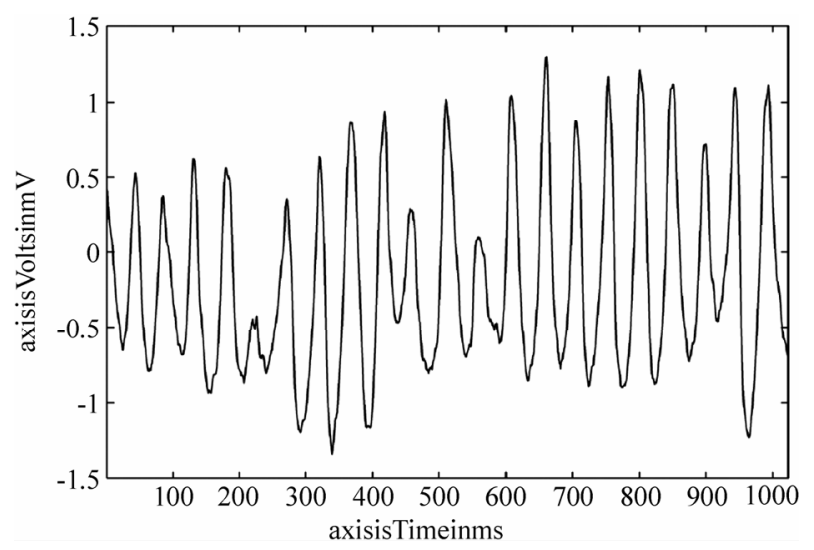

Figure 4. Ventricular flutter.

fibrillation, the heart's ventricles contract in a rapid and unsynchronized way. It is a medical emergency. If this condition continues for more than a few seconds, blood circulation will cease, as evidenced by lack of pulse, blood pressure and respiration, and death will occur [11].

VFL is a tachyarrhythmia characterized by a high ventricular rate with a regular rhythm (Figure 4). The ECG shows large sine wave-like complexes that oscillate in a regular pattern. There is no visible $\mathrm{P}$ wave. $\mathrm{QRS}$ complex and $\mathrm{T}$ wave are merged in regularly occurring undulatory waves with a frequency between 180 and 250 beats per minute. In severe cardiac or systemic disease states, ventricular tachycardia can progress to ventricular flutter, then to ventricular fibrillation.

\section{COMPARISION OF EXISTING ALGORITHMS}

Computer based detection and classification algorithms can achieve good reliability, high degree of accuracy and offer the potential of affordable mass screening for cardiac abnormalities.

Till now, many linear techniques for the detection of ventricular arrhythmias have been developed, such as the probability density function method [12], rate and irregularity analysis, analysis of peaks in the short-term 
autocorrelation function [13], sequential hypothesis testing algorithm [14], correlation waveform analysis, four fast template matching algorithms, VF-filter method [15, 16], spectral analysis [17], and time-frequency analysis [18]. However, these methods exhibit disadvantages, some being too difficult to implement and compute for automated external defibrillators (AED's) and implantable cardioverter defibrillators (ICD's), and some only successful in limited cases. For example, the linear techniques $[13,18]$ using the features of amplitude or frequency have shown their limits, since the amplitude of ECG signal decreases as the VFIB duration increases, and the frequency distribution changes with prolonged VFIB duration.

The VF filter method relies on approximating the VFIB signal as a sinusoidal waveform. The method is equivalent to using a bandpass filter, the central frequency of which is the mean signal frequency. The spectral analysis technique relies on the fact that the VFIB frequency contents are concentrated in the bandwidth $4-7 \mathrm{~Hz}$ [13]. The increased power in this band of frequencies is the major indication of the presence of VFIB. The above spectral analysis technique is applied to stationary signals. However, abrupt changes in the nonstationary ECG signal are spread over the whole frequency range. Important time varying statistical characteristics are lost once the signal has been Fourier transformed.

In recent years time-frequency analysis techniques have proved to be useful in experimental and clinical cardiology. These techniques are needed to fully describe and characterize the various ventricular arrhythmias and facilitate the development of new detection schemes with high correct detection rate, or equivalently, with low false-positive and false-negative performance statistics. The wavelet transforms make possible, the decomposition of a signal into a set of different signals of restricted frequency bands. Wavelet processing can be considered as a set of band pass filters [15]. Moreover, the discrete wavelet transform corresponds to a multiresolution analysis [12] which can reduce the redundancy of each filtered signal so that the processing algorithm can be applied effectively to a small data subset of the original signal.

A classification scheme is developed in which a feed forward neural network is used as a classification tool depending on the distinctive frequency bands of each arrhythmia. The algorithm is applied to ECG signals with ventricular arrhythmia disorders.

\section{WAVELET DECOMPOSITION}

The wavelet transform is a mathematical tool for decomposing a signal into a set of orthogonal waveforms localized both in time and frequency domains. The de- composition produces coefficients, which are functions of the scale (of the wavelet function) and position (shift across the signal).

A wavelet which is limited in time and frequency is called "mother wavelet". Scaling and translation of the mother wavelet gives a family of basis functions called "daughter wavelets".

The wavelet transform of a time signal at any scale is the convolution of the signal and a time-scaled daughter wavelet. Scaling and translation of the mother wavelet is a mechanism by which the transform adapts to the spectral and temporal changes in the signal being analyzed.

The continuous wavelet transform for the signal $\mathrm{x}(\mathrm{t})$ is defined as:

$$
\mathrm{W}(a, b)=\frac{1}{\sqrt{|a|}} \int_{-\infty}^{\infty} x(t) \psi^{*}\left(\frac{t-b}{a}\right) d t
$$

where $\mathrm{a}$ and $\mathrm{b}$ are the dilation (scaling) and translation parameters, respectively. A wide variety of functions can be chosen as mother wavelet $\psi$, provided $\psi(\mathrm{t}) \in \mathrm{L}^{2}$ and

$$
\int_{-\infty}^{\infty} \psi(t) d t=0
$$

The DWT makes possible the decomposition of ECG at various scales into its time-frequency components. In DWT two filters, a Low Pass Filter (LPF) and a High Pass Filter (HPF) are used for the decomposition of ECG at different scales. Each filtered signal is down sampled to reduce the length of the component signals by a factor of two. The output coefficients of LPF are called the Approximation while the output coefficients of HPF are called the Detail. The approximation signal can be sent again to the LPF and HPF of the next level for second level of decomposition; thus we can decompose the signal into its different components at different scale levels.

Figure 5 shows the decomposition process of a signal into many levels. The details of all levels and the approximation of the last level are saved so that the original signal could be reconstructed by the complementary filters. The reconstructed signals at each level are represented by the notations D1, D2, D3 and so on.

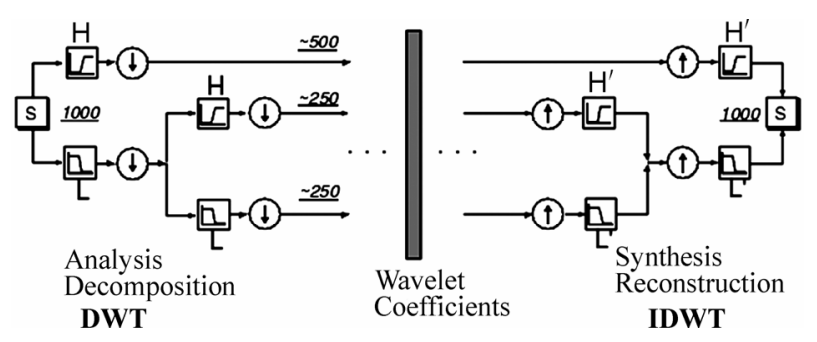

Figure 5. Wavelet decomposition and reconstruction. 


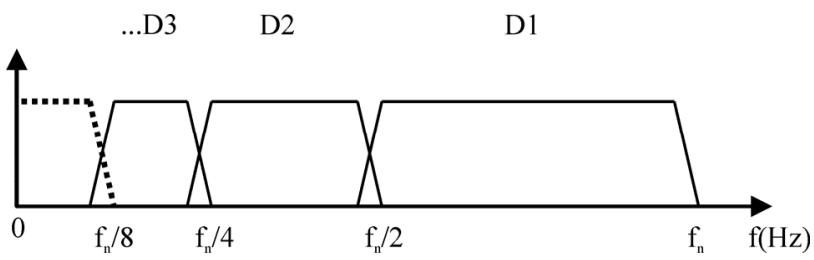

Figure 6. Ideal frequency bands for the various details.

Figure 6 shows the ideal frequency bands for a sampling frequency of 360 samples/second. Depending on the scaling function and the mother wavelet, the actual frequency bands and consequent frequency selectivity of the details are slightly different.

\section{METHODOLOGY}

In this section we present the algorithm which efficiently detects and classifies the various ventricular arrhythmias using wavelet decomposition and neural classification.

\subsection{Description of the Algorithm}

The algorithm first decomposes the ECG signals using wavelet transform. The decomposed signals are fed to the feed forward neural network. The wavelet theory is used as a time-frequency representation technique to provide a method for enhancing the detection of life threatening arrhythmias. It reveals some interesting characteristic features such as low frequency band (0-5 $\mathrm{Hz}$ ) for VFL, two distinct frequency bands $(2-5,6-8 \mathrm{~Hz})$ for VT, and a broad band (2-10 Hz) for VFIB.

A classification scheme is developed in which a neural network is used as a classification tool depending on the above distinctive frequency bands of each arrhythmia.
The algorithm is applied to ECG signals obtained from patients suffering from the arrhythmias, mentioned above.

\subsection{ECG Analysis Using Wavelet Decomposition}

To quantify the differences between the various arrhythmias with the help of the wavelet transform, the densities for different frequency bands are compared. The wavelet transform is performed using Daubechies 4 wavelet since it provides better sensitivity [19].

The algorithm computes the volume underneath the 3D plots of the square modulus of the wavelet transform for several regions of the time-frequency plane. The time-frequency plane is divided into seven bands ranging from 0 to $15 \mathrm{~Hz}$. For sinus rhythm the energy is calculated within the time intervals $T_{1}$ and $T_{2}$ integrated over the whole frequency axis. The time interval $T_{1}$ is determined by the region of QRS complex, and the time interval $\mathrm{T}_{2}$ is determined by the region of the T-wave.

As the wavelet transform is very sensitive to abrupt changes in the time direction, the energy parameter over the given time intervals attains relatively large values for normal subjects. This parameter is referred to as $T_{v}$ and it defines the sum of the energy parameters computed within the intervals $T_{1}$ and $T_{2}$. Although the signals of VFL and VT exhibit a QRS-complex, the parameter value $T_{2}$ for these signals remains relatively small, owing to the absence of abrupt changes in the region of the $\mathrm{T}$-wave. Therefore, the value of $\mathrm{T}_{\mathrm{v}}$ will still be smaller than that of the normal subjects.

The 2D and 3D wavelet transform contours of Normal Sinus Rhythm (NSR), VT and VFL are shown in Figure 7.

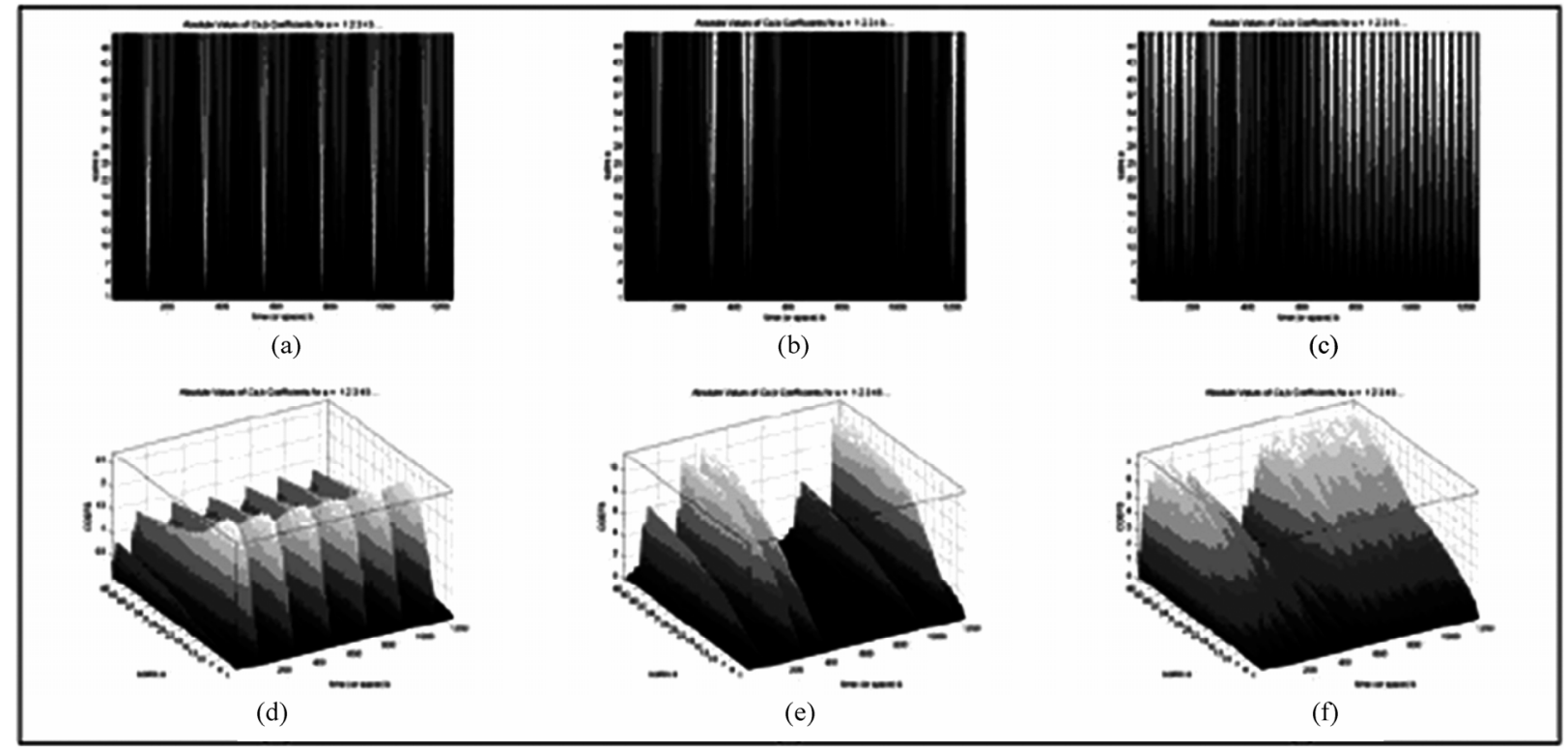

Figure 7. (a) 2D wavelet of NSR, (b) 2D wavelet of VT, (c) 2D wavelet of VFL, (d) 3D wavelet of NSR, (e) 3D wavelet of VT and (f) 3D wavelet of VFL. 


\subsection{Classification of Ventricular Arrhythmias Using Neural Network}

There are quite lot of network topologies with powerful learning strategies which exist to solve nonlinear problems $[20,21,22,23]$. For the classification of Ventricular Arrhythmias, back propagation with momentum is used to train the feed forward neural network [24].

A multilayer feed forward neural network with one layer of hidden $(Z)$ units is used for this problem. The output $(\mathrm{Y})$ units have weights $\mathrm{w}_{\mathrm{jk}}$ and the hidden units have weights $\mathrm{v}_{\mathrm{jk}}$. During the training phase, each output neuron compares its computed activation $\mathrm{y}_{\mathrm{k}}$ with its target value $d_{k}$ to determine the associated Error (E) by using (3) for the pattern with that neuron.

$$
E=\sum_{k=1}^{m}\left(d_{k}-y_{k}\right)^{2}
$$

The weights and biases of the Artificial Neural Network (ANN) are adjusted to minimize the least-square error. The minimization problem is solved by gradient technique, the partial derivatives of $\mathrm{E}$ with respect to weights and biases are calculated using the generalized delta rule. This is achieved by the back propagation of the error.

When using momentum, the neural network is proceeding not in the direction of the gradient, but in the direction of the combination of the current gradient and the previous direction of weight correction. Convergence is sometimes faster if a momentum term is added to the weight update formula. In the back propagation with momentum, the weights for the training step $t+1$ are based on the weights at training steps $t$ and $t-1$. The weight update formulae for back propagation with momentum are given by (4)

$$
\begin{aligned}
& w_{j k}(t+1)=w_{j k}(t)+\alpha \delta_{k} z_{j}+\mu\left[w_{j k}(t)-w_{j k}(t-1)\right] \\
& v_{i j}(t+1)=v_{i j}(t)+\alpha \delta_{j} x_{i}+\mu\left[v_{i j}(t)-v_{i j}(t-1)\right]
\end{aligned}
$$

where the learning factor $\alpha$ and momentum parameter $\mu$ are constrained to be in the range 0 to 1 , exclusive of the end points. The weights and biases are initialized to some random values and updated in each iteration until the network has settled down to a minimum.

The classification of the VT, VFIB and VFL arrhythmia is carried out using a Back-propagation neural network whose input is the energy level calculated by the wavelet transform as described above, the output is a three bit pattern, in which 100 corresponds to VFL, and 010 corresponds to VT, and 001 corresponds to VFIB.

The network has three layers: The input, the output and one hidden layer. The input layer has seven nodes representing the seven different frequency bands from $0-15 \mathrm{~Hz}$. The output layer has three nodes that represent the three different types of arrhythmia signals VFL, VT and VFIB. The hidden layer consists of six nodes for effective size of the network and acceptable efficiency. The learning rate and the momentum term are chosen to be 0.7 and 0.3 respectively.

\subsection{Data}

The MIT-BIH arrhythmia and malignant ventricular arrhythmia databases were used for the analysis [25]. The VT episodes from the records 200, 203, 205, 207, 208, $210,213,214,215,217,221,223,233,106$ etc were taken from the MIT-BIH arrhythmia database. VFIB and VFL episodes were from MIT-BIH malignant ventricular arrhythmia database from the records $418-430,602$, $605,607,609,610,611,612,614,615$ and cu01 - cu35. The data files from arrhythmia database and malignant ventricular arrhythmia database were sampled at 360 samples / second.

\section{RESULTS AND CONCLUSIONS}

Twenty-five signals of VT, ten signals of VFL and five signals of VFIB are used for training. The learning process took approximately 1000 iterations to converge with classification error of 0.001 . Thirty signals of VT, fifteen signals of VFL and four signals of VFIB are selected as test set. For decomposition of ECG signal Daubechies 4 wavelet is used.

In medical statistics, few parameters are important to evaluate the performance of the algorithm. These parameters are sensitivity $(\mathrm{Se})$ and positive predictivity $(+\mathrm{P})$ which can be computed using (5) and (6).

$$
\begin{aligned}
& \mathrm{Se}=\frac{\mathrm{TP}}{\mathrm{TP}+\mathrm{FN}} \\
& +\mathrm{P}=\frac{\mathrm{TP}}{\mathrm{TP}+\mathrm{FP}}
\end{aligned}
$$

where TP is True Positive, FP is False Positive and FN is False Negative.

Classification results of testing data sets using Feed Forward Neural Network with BP are shown in Table 1. Sensitivity and Positive predictivity for the various ventricular arrhythmias are shown in Table 2 . The results

\begin{tabular}{|c|c|c|c|c|}
\hline \multirow{2}{*}{$\begin{array}{l}\text { Actual Ventricular } \\
\text { Arrhythmia }\end{array}$} & \multicolumn{3}{|c|}{ Detected Ventricular Arrhythmia } & \multirow{2}{*}{$\begin{array}{c}\text { Total } \\
49\end{array}$} \\
\hline & VT & VFL & VFIB & \\
\hline VT & 30 & 0 & 0 & 30 \\
\hline VFL & 1 & 13 & 1 & 15 \\
\hline VFIB & 0 & 0 & 4 & 4 \\
\hline
\end{tabular}
show that the BP algorithm is reliable in the classification of all the three types of ventricular arrhythmias.

Table 1. A comparison between the actual and detected ventricular arrhythmias in terms of the number of patterns. 
Table 2. Sensitivity and positive predictivity for the testing set.

\begin{tabular}{cccccc}
\hline $\begin{array}{c}\text { Ventricular Ar- } \\
\text { rhythmia }\end{array}$ & TP & FP & FN & $\begin{array}{c}\text { Positive Pre- } \\
\text { dictivity (\%) }\end{array}$ & Sensitivity (\%) \\
\hline VT & 30 & 1 & 0 & 96.77 & 100 \\
VFL & 13 & 0 & 2 & 100 & 86.67 \\
VFIB & 4 & 1 & 0 & 80 & 100 \\
\hline
\end{tabular}

Table 1 shows that the BP network misclassified the proper arrhythmia in some cases, one VFL case was classified as VFIB, this is because the energy level in the frequency bands is high and common between VFL and VFIB. The algorithm is able to classify VT and VFIB with $100 \%$ sensitivity. The positive predictivity for VFL episodes is $100 \%$. The algorithm is reliable by providing the overall sensitivity of $95.56 \%$ and the overall positive predictivity of $92.26 \%$. The algorithm can be validated using more number of ECG samples.

\section{REFERENCES}

[1] K. Anant, F. Dowla, and G. Rodrigue, (1995) Vector quantization of ECG wavelet coefficients, IEEE Signal Processing Letters, 2(7).

[2] M. Vetterli, (1992) Wavelets and filter banks: Theory and design, IEEE Transactions on Signal Processing, 22072232.

[3] R. M. Rao and A. S. Bopardikar, (1998) Wavelet transforms: Introduction to theory and applications, Addison Wesley Longman.

[4] L. Khadra, A. S. Al-Fahoum, and H. Al-Nashash, (1997) Detection of life threatening cardiac arrhythmia using the wavelet transformation, Med. Biol. Eng. Comput., 35, 626-632.

[5] P. S. Addison, J. N. Watson, G. R. Clegg, M. Holzer, F. Sterz, and C. E. Robertson, (2000) Evaluating arrhythmias in ECG signals using wavelet transforms, IEEE Engineering in Medicine and Biology Magazine, 19, 104 109.

[6] H. A. N. Dinh, D. K. Kumar, N. D. Pah, and P. Burton, (2001) Wavelets for QRS detection, Proceedings of the $23^{\text {rd }}$ Annual Conference, IEEE EMS, Istanbul, Turkey, 35-38.

[7] S. Kadambe, R. Murray, and G. F. Boudreaux-Bartels, (1999) Wavelet transform based QRS complex detector, IEEE Transaction on Biomedical Engineering, 46(7), 838-848.

[8] I. Romero, L. Serrano, and Ayesta, (2001) ECG frequency domain features extraction: A new characteristic for arrhythmias classification, Conference of the IEEE Engineering in Medicine and Biology Society.

[9] S. M. Szilagyi and L. Szilagyi, (2000) Wavelet transform and neural network based adaptive filtering for QRS de- tection, Proceedings of World Congress on Medical Physics and Biomedical Engineering, Chicago, USA.

[10] D. E. Rumelhart, G. E. Hinton, and R. J Williams, (1986) Learning representations by back-propagation errors, Nature.

[11] V. X. Afonso and W. J. Tompkins, (1995) Detecting ventricular fibrillation, IEEE Eng. Boil., 152-159.

[12] A. Langer, M. S. Heilman, and M. M. Mower, (1976) Considerations in the development of the automatic implantable defibrillator, Medical Instrumentation, 10(3), 163-167.

[13] S. Chen, N. V. Thakor, and M. M. Mover, Ventricular fibrillation detection by a regression test on the autocorrelation function, Med. Biol. Eng. Comput., 25(3), 241249.

[14] S. W. Chen, P. W. Clarkson, and Q. Fan, (1996) A robust detection algorithm for cardiac arrhythmia classification, IEEE Transactions on Biomedical Engineering, 43, $1120-1125$

[15] R. H. Clayton, A. Murray, and R.W. F. Campbell, (1994) Recognition of ventricular fibrillation using neural networks, Med. Bio. Eng. Comp., 32, 217-220.

[16] S. Kuo and R. Dillman, (1978) Computer detection of ventricular fibrillation, Computer Cardiology, 347-349.

[17] S. Barro, R. Ruiz, D. Cabello, and J. Mira, (1989) Algorithmic sequential decision-making in the frequency domain for life threatening ventricular arrhythmias and imitative artifacts: A diagnostic system, Journal on Biomedical Engineering, 11(4), 320-328.

[18] V. X. Afonso, W. J. Tompkins, T. Q. Nguyen, and S. Luo, (1999) ECG beat detection using filter banks, IEEE Transactions on Biomedical Engineering, 46(2), 192202 ,

[19] G. Selvakumar, B. K. Bhoopathy, and R. B. Chidhambara, (2007) Wavelet decomposition for detection and classification of critical ECG arrhythmias, Proc. of the 8th WSEAS Int. Conf. on Mathematics And Computers in Biology and Chemistry, Vancouver, Canada.

[20] G. Bortalan and J. L. Willems, (1993) Diagnostic ECG classification based on neural networks, Journal of Electrocardiology, 26, 75-79.

[21] Z. Dokur, T. Olmez, and E. Yazgan, (1997) Detection of ECG waveforms by neural networks, Journal on Medical Engineering and Physics, 19(8), 738-741.

[22] L. Edenbrandt, B. Heden, and O. Pahlm, (1993) Neural networks for analysis of ECG complexes, Journal of Electrocardiology, 26, 74.

[23] R. Silipo and C. Marchesi, (1998) Artificial neural networks for automatic ECG analysis, IEEE Trans. on Signal Processing, 46.

[24] A. S. Al-Fahoum and I. Howitt, (1999) Combined wavelet transformation and radial basis neural networks for classifying life threatening cardiac arrhythmias, Med. Biol. Eng. Comput., 37, 566-573.

[25] MIT-BIH (http://www.physionet.org) 